\title{
Ultra-short solitons and kinetic effects in nonlinear metamaterials
}

\author{
Mattias Marklund, ${ }^{1,2}$ Padma K. Shukla, ${ }^{3,2}$ and Lennart Stenflo ${ }^{1}$ \\ ${ }^{1}$ Department of Physics, Umeå University, SE-901 87 Umeå, Sweden \\ ${ }^{2}$ Centre for Fundamental Physics, Rutherford Appleton Laboratory, Chilton, Didcot, Oxfordshire, UK \\ ${ }^{3}$ Institut für Theoretische Physik IV, Fakultät für Physik und Astronomie, \\ Ruhr-Universität Bochum, D-44780 Bochum, Germany
}

(Dated: January 23, 2006)

\begin{abstract}
We present a stability analysis of a modified nonlinear Schrödinger equation describing the propagation of ultra-short pulses in negative refractive index media. Moreover, using methods of quantum statistics, we derive a kinetic equation for the pulses, making it possible to analyze and describe partial coherence in metamaterials. It is shown that a novel short pulse soliton, which is found analytically, can propagate in the medium.
\end{abstract}

PACS numbers: 42.25.Bs, 42.25.Gy, 78.20.Ci

As early as in 1945, Mandelstam studied the electromagnetic properties of materials exhibiting a negative index of refraction [1, 2]. The concepts of negative permittivity $\varepsilon$ and permeability $\mu$ and the consequences of negative group velocity were moreover given attention by Pafomov [3] and Agranovich and Ginzburg [4], and have since then been discussed by several authors, e.g. [5, 6, 7, 8]. Recently, such materials have been produced [9, 10], and experimental verifications of previous theoretical issues are currently attracting much interest (see, e.g. [11, 12] for a review). From a sharp resonance in the material response to the applied external field, one may obtain negative $\varepsilon$ and $\mu$. The normal procedure for obtaining negative-index-of-refraction materials is to put together two structured materials that both have negative permittivity and negative permeability, such that the resulting composite material has a negative refractive index 12]. Since such materials may have a nonlinear response as well, a nonlinear metamaterial can be constructed 13, 14]

In the present Brief Report, we shall consider ultra-short electromagnetic pulses in left-handed materials [14]. We investigate the stability properties of a pulse, and show that the metamaterial can influence its dynamics in significant ways. Moreover, the effects of partial pulse coherence is studied using the Wigner function approach. It is also shown that the dynamical equation for modulated short pulses admits a novel localized envelope soliton solution, which should be experimentally verified.

Given the electric field $E(z, t) \exp \left(i k_{0} z-i \omega_{0} t\right)$ of a short electromagnetic pulse, the evolution of the pulse envelope $E$ in the slowly varying envelope limit, i.e. $k_{0} \gg\left(\partial_{z}-2\left(\partial k_{0} / \partial \omega_{0}\right) \partial_{t}\right)$, is governed by (e.g. [14])

$$
i \frac{\partial E}{\partial z}+\frac{\alpha}{2} \frac{\partial^{2} E}{\partial t^{2}}+\kappa_{1}\left(1-\kappa_{2} I\right) I E-i \sigma \frac{\partial}{\partial t}(I E)=0,
$$

where we have introduced the parameters $\alpha=-\left(\partial^{2} k_{0} / \partial \omega_{0}^{2}\right), \kappa_{1}=\beta \mu \chi^{(3)} / 2 n, \kappa_{2}=\mu \chi^{(3)} / 4 n^{2}$, and $\sigma=$ $\chi^{(3)}\left[\mu / 2 V_{g} n^{2}-(\gamma+\mu) / 2 n\right]$. Moreover, $\beta=2 \pi \omega_{0} / \omega_{p}, \omega_{p}$ is the plasma frequency, $\chi^{(3)}$ is the third order susceptibility of the medium, $-\alpha$ is the group velocity dispersion (GVD), $V_{g}=\left(\partial k_{0} / \partial \omega_{0}\right)^{-1}$ is the group velocity, $\mu$ is the magnetic permeability, $n$ is the refractive index, $\gamma=\partial\left(\omega_{0} n\right) / \partial \omega_{0}$, and the intensity is given by $I=|E|^{2}$.

There is a stationary solution $E(t, z)=E_{0} \exp (i \Delta k z)$ of Eq. (11), corresponding to a nonlinear phase shift

$$
\Delta k=\kappa_{1}\left(1-\kappa_{2} I_{0}\right) I_{0},
$$

where $I_{0}=E_{0}^{2}$. Linearizing around this stationary solution, according to $E(t, z)=\left[E_{0}+E_{1}(t, z)\right] \exp (i \Delta k z)$, where $\left|E_{1}\right| \ll E_{0}$, Eq. (11) gives

$$
i \frac{\partial E_{1}}{\partial z}+\frac{\alpha}{2} \frac{\partial^{2} E_{1}}{\partial t^{2}}+\kappa_{1}\left(1-\kappa_{2} I_{0}\right) I_{0}\left(E_{1}+E_{1}^{*}\right)-i \sigma I_{0} \frac{\partial}{\partial t}\left(2 E_{1}+E_{1}^{*}\right)=0 .
$$

By making the ansatz $E_{1}=E_{1 r}+i E_{1 i}$, and assuming a harmonic dependence in the form $E_{1 r}, E_{1 i} \propto \exp (i K z-i \Omega t)$, we can split Eq. (3) into its real and imaginary parts, and obtain the dispersion relation

$$
K=-2 \sigma \Omega I_{0} \pm \Omega\left[\left(\alpha \kappa_{1} \kappa_{2}+\sigma^{2}\right) I_{0}^{2}-\alpha \kappa_{1} I_{0}+\frac{\alpha^{2}}{4} \Omega^{2}\right]^{1 / 2}
$$


Letting $K=-2 \sigma \Omega I_{0}-i \Gamma$, where $\Gamma>0$, we thus have the modulational instability growth rate

$$
\Gamma=\Omega\left[\alpha \kappa_{1} I_{0}-\left(\alpha \kappa_{1} \kappa_{2}+\sigma^{2}\right) I_{0}^{2}-\frac{\alpha^{2}}{4} \Omega^{2}\right]^{1 / 2}
$$

The effects of the material parameters $\alpha, \kappa_{1}, \kappa_{2}$, and $\sigma$ on the modulational instability growth rate (5) can be divided into a number of cases. We note that in the coherent case the effect of the derivative nonlinearity is always to suppress the modulational instability.

First, in the case of positive GVD, i.e. $\alpha<0$, there is no growth in a focusing medium $\left(\chi^{(3)}>0 ; \kappa_{1}>0\right)$ if the higher order nonlinearities through $\kappa_{2}$ are neglected. If the quintic nonlinearities are taken into account for focusing media, we see from (5) that a positive growth rate is possible, given that $\kappa_{1} \kappa_{2}>0$. Since $\kappa_{1} / \kappa_{2}=2 \beta n$, we will thus have growth for normal and focusing media, where both the third order susceptibility and the refractive index are positive. In focusing metamaterials with positive GVD this is no longer possible, and the modulational instability thus disappears. If we instead have a defocusing medium, i.e. $\chi^{(3)}<0$, this implies $\kappa_{1}<0$, and $\alpha \kappa_{1}>0$. Thus, growth is possible even if $\kappa_{2}=0$. For normal bulk media, $\alpha \kappa_{1} \kappa_{2}<0$ which increases the modulational instability growth rate. However, for left-handed metamaterials we have $\alpha \kappa_{1} \kappa_{2}>0$, which implies a suppression of the modulational instability.

Secondly, there is the case of negative GVD, so that $\alpha>0$. If we have a focusing medium, so that $\chi^{(3)}>0$ and $\kappa_{1}>0$, the effect of a metamaterial in which $\kappa_{2}<0$, is to increase the growth rate (5), while normal media tend to inhibit the modulational instability. However, if the medium is defocusing, the coherent modulational instability is not possible in a normal medium, while in left-handed metamaterials we can obtain a positive growth rate from (5).

Thus, we see from the cases above that the interplay between the material coefficients is complex, and requires careful consideration when studying the stability properties of waves in metamaterials.

In order to take the effects of partial incoherence into account, we define the space-time correlation function for the electric field as $\widetilde{F}\left(z_{+}, z_{-}, t_{+}, t_{-}\right)=\left\langle E^{*}\left(z_{+}, t_{+}\right) E\left(z_{-}, t_{-}\right)\right\rangle$, where $z_{ \pm}=z \pm \zeta / 2, t_{ \pm}=t \pm \tau / 2$, and the angular brackets denote the ensemble average. The two-point correlation function for the field can be used to analyze, for example, self-trapping of light and incoherent soliton propagation in optical structures and nonlinear fibers [15, 16, 17]. Here we will use the Fourier transform of the correlation function, as this will give a generalized kinetic description for the quasi particles. The Wigner distribution function of the pulse is given by [18, 19]

$$
F(z, t, k, \omega)=\frac{1}{(2 \pi)^{2}} \int d \zeta d \tau e^{i(k \zeta-\omega \tau)} \widetilde{F}\left(z_{+}, z_{-}, t_{+}, t_{-}\right)
$$

with the inverse $\widetilde{F}\left(z_{+}, z_{-}, t_{+}, t_{-}\right)=\int d k d \omega e^{-i(k \zeta-\omega \tau)} F(z, t, k, \omega)$ such that

$$
I(z, t)=\int d k d \omega F(z, t, k, \omega)
$$

Thus, from Eq. (11) the evolution equation for the Wigner function (6) corresponding to the envelope field $E$ is (see also Ref. [20])

$$
\begin{aligned}
& \alpha \omega \partial_{t} F-\partial_{z} F+2 \kappa_{1}\left(1-\kappa_{2} I\right) I \sin \left(\frac{1}{2} \overleftarrow{\partial_{t}} \vec{\partial}_{\omega}\right) F \\
& \quad+\sigma\left\{\partial_{t}\left[I \cos \left(\frac{1}{2} \overleftarrow{\partial_{t}} \vec{\partial}_{\omega}\right) F\right]-2 \omega I \sin \left(\frac{1}{2} \overleftarrow{\partial_{t}} \vec{\partial}_{\omega}\right) F\right\}=0
\end{aligned}
$$

where we have made a Wigner transformation over the time domain. Here the arrows denote the direction of operation, and the operator functions are defined in terms of their respective Taylor expansion. The system of equations (7) and (8) determines the evolution of short partially incoherent pulses in nonlinear meta-materials.

In the low-frequency limit, we retain only the lowest order terms in the sin and cos operators in Eq. (8), to obtain the Vlasov-like equation

$$
\alpha \omega \partial_{t} F-\partial_{z} F+\kappa_{1} \partial_{t}\left[\left(1-\kappa_{2} I\right) I\right] \partial_{\omega} F+\sigma\left[\frac{1}{2} \partial_{t}(I F)-\omega\left(\partial_{t} I\right) \partial_{\omega} F\right]=0
$$


In order to analyse the modulational instability and the effects of the terms due to nonzero $\sigma$ and $\kappa_{2}$, we write $F(z, t, \omega)=F_{0}(\omega)+F_{1}(\omega) \exp (i K z-i \Omega t)+$ c.c., where c.c. denotes the complex conjugate, and $\left|F_{1}\right| \ll F_{0}$. Expanding Eq. (8) in terms of this ansatz, and using Eq. (7), we obtain

$$
\begin{aligned}
1= & \frac{1}{\alpha \Omega} \int_{-\infty}^{\infty} d \omega\left\{\left[\kappa_{1}\left(1-\kappa_{2} I_{0}\right)-\sigma(\omega+\Omega / 2)\right] F_{0}(\omega-\Omega / 2)\right. \\
& \left.-\left[\kappa_{1}\left(1-\kappa_{2} I_{0}\right)-\sigma(\omega-\Omega / 2)\right] F_{0}(\omega+\Omega / 2)\right\}\left[\omega+\left(K+\sigma \Omega I_{0}\right) / \alpha \Omega\right]^{-1},
\end{aligned}
$$

where $I_{0}=\int d \omega F_{0}(\omega)$. Equation (10) represents the NDR for a short electromagnetic pulse, where the pulse may have spectral broadening and partial incoherence.

In the case of a mono-energetic pulse, we have $F_{0}(\omega)=I_{0} \delta\left(\omega-\Omega_{0}\right)$, where $\Omega_{0}$ corresponds to a frequency shift of the background plane wave solution, and the NDR (10) gives

$$
K=-\left(2 \sigma I_{0}+\alpha \Omega_{0}\right) \Omega \pm \Omega\left[\left(\alpha \kappa_{1} \kappa_{2}+\sigma^{2}\right) I_{0}^{2}-\alpha \kappa_{1} I_{0}+\frac{\alpha^{2}}{4} \Omega^{2}+\alpha \sigma \Omega_{0} I_{0}\right]^{1 / 2}
$$

In practice however, the wave envelope will always suffer from perturbations due to various noise sources, e.g. thermal spread in the material. A noisy environment may cause the pulse field to attain a random component in its phase. Thus, if the phase $\varphi(x)$ of the electric field varies stochastically, such that the ensemble average of the phase satisfies [21]

$$
\langle\exp [-i \varphi(t+\tau / 2)] \exp [i \varphi(t-\tau / 2)]\rangle=\exp \left(-\Omega_{T}|\tau|\right)
$$

the background Wigner distribution is given by the Lorentzian spectrum

$$
F_{0}(\omega)=\frac{I_{0}}{\pi} \frac{\Omega_{T}}{\left(\omega-\Omega_{0}\right)^{2}+\Omega_{T}^{2}}
$$

where $\Omega_{T}$ corresponds to the width of the spectrum. Then, the NDR (10) takes the form

$$
1=-I_{0} \Omega \frac{2 \sigma\left[K+\sigma I_{0} \Omega+\frac{\alpha}{2} \Omega\left(\Omega_{0}-i \Omega_{T}\right)\right]+\alpha \kappa_{1}\left(1-\kappa_{2} I_{0}\right) \Omega}{\left(K+\sigma I_{0} \Omega+\Omega_{0}-i \Omega_{T}\right)^{2}-\frac{\alpha^{2}}{4} \Omega^{4}}
$$

which has the solution

$$
K=-\left[2 \sigma I_{0}+\alpha\left(\Omega_{0}-i \Omega_{T}\right)\right] \Omega \pm \Omega\left[\left(\alpha \kappa_{1} \kappa_{2}+\sigma^{2}\right) I_{0}^{2}-\alpha \kappa_{1} I_{0}+\frac{\alpha^{2}}{4} \Omega^{2}+\alpha \sigma\left(\Omega_{0}-i \Omega_{T}\right) I_{0}\right]^{1 / 2} .
$$

This solution generalizes (11) to the case of a random phase background envelope field. The expression (15) clearly shows that the width gives a nontrivial contribution to the NDR. We note that when $\sigma=0$, we may define the growth rate $\kappa$ according to $K=-\alpha \Omega_{0} \Omega-i \Gamma$, and the width $\Omega_{T}$ then gives rise to a Landau like damping due to (15).

We may normalize the dispersion relation (15), assuming the GVD to be negative, i.e. $\alpha>0$, according to $I_{0} \rightarrow \kappa_{1} I_{0}$, $\Omega_{T} \rightarrow \sqrt{\alpha} \Omega_{T}, \Omega \rightarrow \sqrt{\alpha} \Omega, \sigma \rightarrow \sigma /\left(\kappa_{1} \sqrt{\alpha}\right)$, and $\kappa=\kappa_{2} / \kappa_{1}=1 / 2 n \beta$. The dispersion relation (15) then reads

$$
K=-\left(2 \sigma I_{0}+\Omega_{0}-i \Omega_{T}\right) \Omega \pm \Omega\left[\left(\kappa+\sigma^{2}\right) I_{0}^{2}-I_{0}+\frac{1}{4} \Omega^{2}+\sigma\left(\Omega_{0}-i \Omega_{T}\right) I_{0}\right]^{1 / 2} .
$$

Letting $K=\operatorname{Re} K-i \Gamma$, where Re denotes the real part, we can solve Eq. (16) for the growth rate $\Gamma$. Thus, the general perturbation exhibits oscillatory growth or damping. The effect of a finite $\Omega_{T}$ is in general to reduce the growth rate, but it may in certain cases broaden the spectral instability region. We note that $\sigma$ and $\kappa$ have both positive and negative values, depending on whether the material is a normal bulk medium, or if it allows for a negative index of refraction. For the case of a normal bulk material, we have $\kappa>0$, and the higher order nonlinear correction will thus reduce the growth rate [14]. However, for metamaterials we have $\kappa<0$, altering the dynamics of Eq. (11) in nontrivial ways. Thus, for metamaterials, the modulational instability growth rate will differ in measurable ways from the case of normal bulk media. We also note that for the modulational instability growth rate, the sign of $\sigma$ is irrelevant. Since the full solution is symmetric with respect to $\sigma \Omega$, requiring $\Omega>0$ makes the choice of sign for $\sigma$ unimportant in this 
case. In Fig. 1 we have plotted the growth rate for both positive and negative values of $\kappa$, so that the difference can clearly be seen. Thus, it is reasonable to conclude that soliton formation and filamentation of ultra-short pulses can be much more effective in metamaterials as compared to normal bulk media.

In fact, using the techniques of previous work (e.g. [22, 23, 24, 25, 26, 27, 28, 29]), one can obtain a novel exact analytical solution to Eq. (1) representing bright solitons in the form

$$
E(t, z)=\frac{E_{0} \exp [i(K z-\Omega t+\varphi)]}{\sqrt{a+\cosh ^{2}\left[k\left(v t-z+z_{0}\right)\right]}} \exp \left\{i \delta \operatorname{arctanh}\left[\left(\frac{a}{1+a}\right)^{1 / 2} \tanh \left[k\left(v t-z+z_{0}\right)\right]\right]\right\}
$$

where $\varphi$ is a constant phase and $z_{0}$ is a spatial displacement, and we require $a \geq 0$. Furthermore

$$
\delta=\frac{3 \sigma E_{0}^{2}}{2 \alpha v k \sqrt{a(1+a)}}, \quad \Omega=-\frac{1}{\alpha v}, \quad a=\frac{E_{0}^{2}\left(\sigma+\alpha \kappa_{1} v\right)}{2 \alpha^{2} k^{2} v^{3}}-\frac{1}{2}, \text { and } \quad E_{0}^{4}=\frac{3 \alpha^{4} k^{4} v^{6}}{\Lambda}
$$

where $\Lambda=-\alpha \kappa_{1} v\left[8 \kappa_{2} \alpha^{2} k^{2} v^{3}-3\left(\kappa_{1} \alpha v+2 \sigma\right)\right]+3 \sigma^{2}\left(1+\alpha^{2} k^{2} v^{4}\right)$. For $a \rightarrow 0$ we obtain the solitary waves found in Ref. 30, if the material parameters satisfy $\sigma^{2}=8 \alpha \kappa_{1} \kappa_{2} / 3$, while $a \gg 1$ corresponds to a flat-top wave form (see Fig. 2). We also not that a nonzero value of $\sigma$ requires a travelling soliton solution (see Fig. 2).

The width of the pulse is characterized by $1 / k$. It is however limited by the slowly varying envelope approximation used in deducing the basic Eq. (1), and limits how short the pulse can be [31]. This means that the parameter $k$ has to be much smaller than the pump wave number $k_{0}$.

The issue of stability of solitary solutions is an interesting but complex topic (see e.g. 32, 33]). Bright soliton solutions to the one-dimensional cubic nonlinear Schrödinger equation are known to be unstable to transverse perturbations, but it is also known that competing nonlinearities, occuring in cubic-quintic systems, can stabilize soliton solutions 34]. Our equation (11) contains such cubic-quintic nonlinearites, as well as a derivative nonlinearity. It is therefore not unreasonable to conjecture that the solution (17) is stable. However, in order to strictly answer the question of stability of (17) much further research is needed.

To summarize, we have examined the modulational instability and localization of an ultra-short electromagnetic pulse that is governed by a modified nonlinear Schrödinger equation, which is also valid for negative refractive index media. We have considered both coherent and incoherent pulses and derived nonlinear dispersion relations that depict the growth rates of the modulational instabilities. The effect of partial coherence of the electromagnetic pulse is to reduce the instability growth rate. Furthermore, we have found that modulationally unstable pulses can appear as a new type of light envelope solitons whose profiles are found analytically and analyzed numerically. Localized light pulses can propagate without distortion and should be observable in metamaterials which have negative index of refraction.

\section{Acknowledgments}

This research was supported by the Swedish Research Council through the contract No. 621-2004-3217.

[1] L.I. Mandelstam, Zh. Eksp. Teor. Fiz. 15, 475 (1945).

[2] L.I. Mandelstam, The 4th Lecture of L.I. Mandelstam given at Moscow State University (05/05/1944), Collection of Scientific Works, Vol. 2 (1994) Nauka, Moscow (in Russian).

[3] V. E. Pafomov, Sov. Phys. JETP 36, 1321 (1959).

[4] V. M. Agranovich and V. L. Ginzburg, Spatial Dispersion in Crystal Optics and the Theory of Excitons (John Wiley \& Sons, London, 1966); See also V. M. Agranovich and V. L. Ginzburg, Crystal Optics with Spatial Dispersion of Excitons (Springer, Berlin, 1984), p. 252.

[5] V. G. Veselago, Sov. Phys. Usp. 10, 509 (1968).

[6] V.M. Agranovich et al., Phys. Rev. B 69, 165112 (2004).

[7] V.M. Agranovich et al., J. Luminescence 110, 167 (2004).

[8] Y. Zhang and A. Mascarehnas, Mod. Phys. Lett. B 19, 21 (2005).

[9] D. R. Smith and N. Kroll, Phys. Rev. Lett. 85, 2933 (2000); D. R. Smith et al., ibid. 84, 4184 (2000). 
[10] R. A. Shelby, D. R. Smith and S. Schultz, Science 292, 77 (2001); C. G. Parazzoli et al., Phys. Rev. Lett. 90, 107401 (2003); A. A. Houck et al., ibid. 90, 137401 (2003); P. Parini et al., Nature (London) 426, 404 (2003).

[11] J. B. Pendry and D. R. Smith, Phys. Today 57, 37 (2004); J. B. Pendry, Contemporary Phys. 45, 191 (2004).

[12] S. Anantha Ramakrishna, Rep. Prog. Phys. 68, 449 (2005).

[13] G. D'Aguanno, N. Mattiucci, M. Scalora, and M. J. Bloemer, Phys. Rev. Lett. 93, 213902 (2004).

[14] M. Scalora et al., Phys. Rev. Lett. 95, 013902 (2005).

[15] M. Mitchell et al., Phys. Rev. Lett. 77, 490 (1996); M. Mitchell and M. Segev, Nature 387, 880 (1997).

[16] D. N. Christodoulides et al., Phys. Rev. Lett. 78, 646 (1997); M. Mitchell et al., Phys. Rev. Lett. 79, 4990 (1997); D. N. Christodoulides et al., Phys. Rev. Lett. 80, 2310 (1998).

[17] W. Królikowski, O. Bang, and J. Wyller, Phys. Rev. E 70, 036617 (2004).

[18] E. Wigner, Phys. Rev. 40, 749 (1932).

[19] J.T. Mendonça, Theory of Photon Acceleration (IOP Publishing, Bristol, 2001).

[20] I.M. Besieris and F.D. Tappert, J. Math. Phys. 17, 734 (1976).

[21] R. Loudon, The Quantum Theory of Light (Oxford University Press, Oxford, 2000).

[22] N. R. Pereira and L. Stenflo, Phys. Fluids 20, 1733 (1977).

[23] B. A. Malomed, Physica D 29, 155 (1987).

[24] B. A. Malomed, Phys. Rev. A 44, 6954 (1991).

[25] S. Kishiba, S. Toh, and T. Kawahara, Physica D 54, 43 (1991).

[26] R. Conte and M. Musette, Physica D 69, 1 (1993).

[27] V. V. Afanasjev, Opt. Lett. 20, 704 (1995).

[28] B. A. Malomed et al., Physica Scripta T82, 36 (1999).

[29] V. S. Filho et al.,Phys. Rev. A 63, 053603 (2001).

[30] R. Radhakrishnan, A. Kundu, and M. Lakshmanan, Phys. Rev. E 60, 3314 (1999).

[31] K. E. Oughstun and H. Xiao, Phys. Rev. Lett. 78, 642 (1997).

[32] L.-C. Crasovan et al., Phys. Rev. E 62, 1322 (2000).

[33] B. A. Malomed et al., Phys. Lett. 274, 111 (2000).

[34] M. Quiroga-Teixeiro and H. Michinel, J. Opt. Soc. Am. B 14, 2004 (1997). 


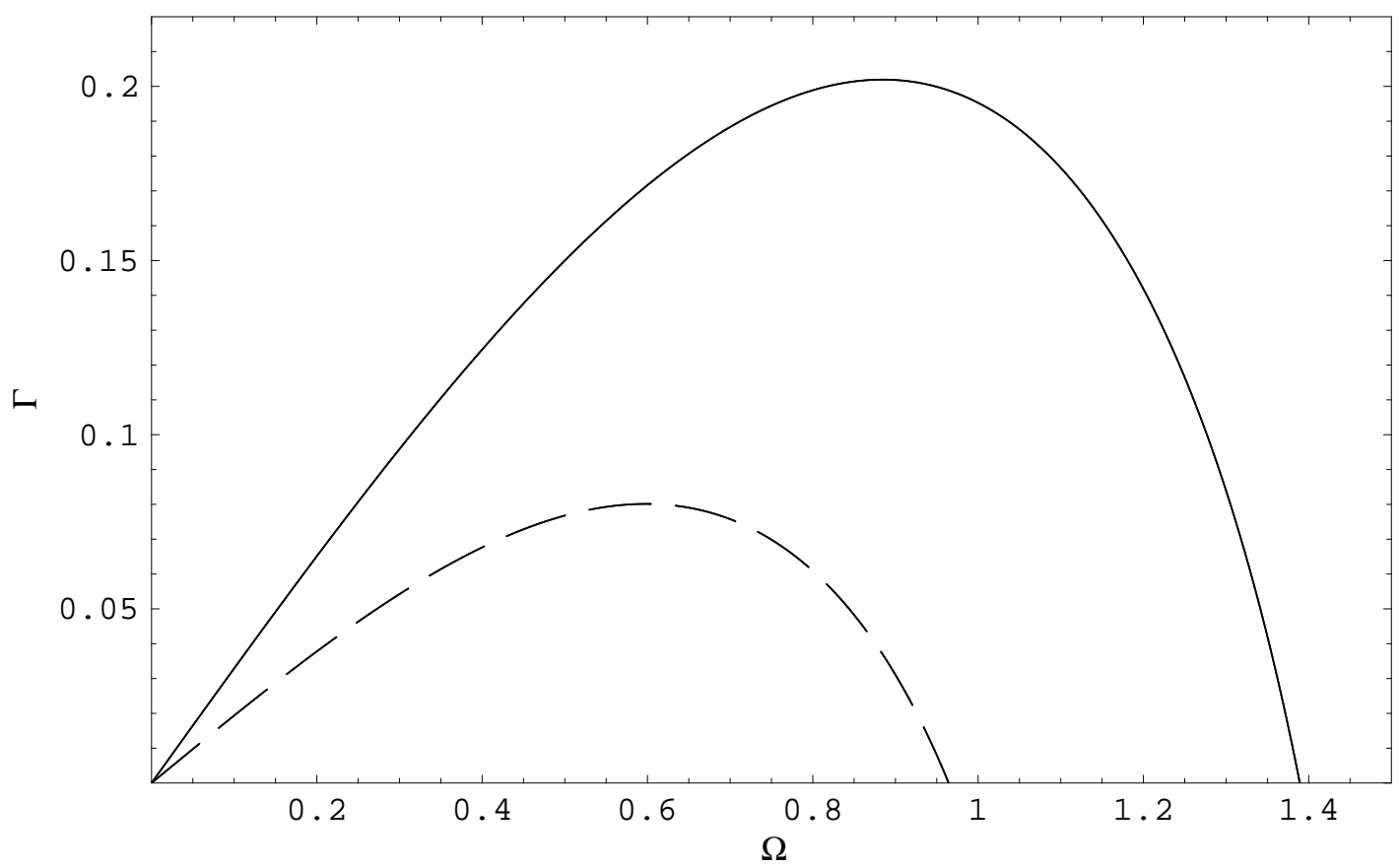

FIG. 1: The modulational instability growth rate plotted for $I_{0}=0.5$, using $\sigma=0.5, \Omega_{T}=0.1$, and $\kappa=-0.5$ (upper curve) and $\kappa=0.5$ (lower curve). We have here normalized the growth rate by $\kappa_{1} I_{0}$ and the frequency by $\sqrt{\left|\kappa_{1}\right| I_{0} / \alpha}$, and correspondingly for the parameters in Eq. (15), thus giving dimensionless quantities. The larger growth rate for metamaterials is clearly seen.

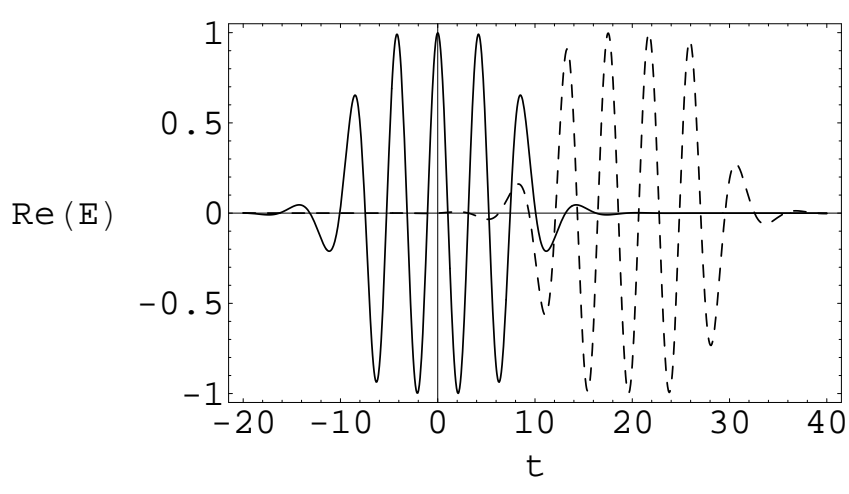

(a)

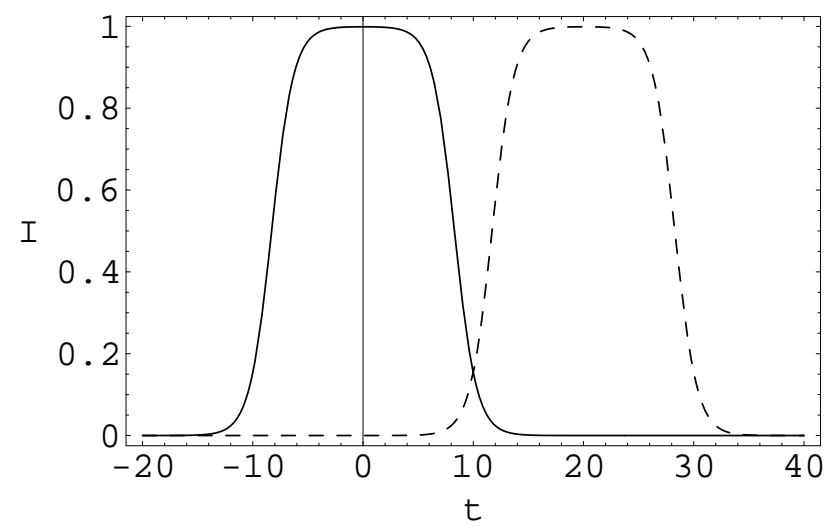

(b)

FIG. 2: The characteristic features of the soliton solution (17). The real part of the solution (17) is plotted in panel (a), while the intensity $I=|E|^{2}$ is plotted in panel (b), both for two different values of $z$. We have normalized the solution so that $E \rightarrow \sqrt{a} E / E_{0}$, while time is normalized by $\sqrt{\left|\kappa_{1}\right| E_{0}^{2} / \alpha}$. 